\title{
Introduction to Non-Statistical Natural Language Processing
}

\author{
Graeme Hirst \\ University of Toronto
}

\section{Tutorial Outline}

1. Symbolic NLP

2. Words, lexicons, morphology

3. Syntax, grammars

4. Parsing algorithms

5. Semantic representations

6. Semantic analysis

7. Generating language from meaning

8. Discourse structure and relations

9. Language in use, implicature, presupposition

\begin{abstract}
Many problems in natural language processing are best understood and approached by symbolic rather than statistical methods, especially problems in using the structure and meaning of sentences, paragraphs, texts, and dialogues. This tutorial will introduce symbolic methods in syntactic analysis, semantic structures and the representation of meaning, discourse and dialogue structure, and the pragmatics of language in use.
\end{abstract}

